\title{
Endoscopic stenting in grade III pancreatic injury according to the American Association for the Surgery of Trauma - a systematic review of case reports and case series
}

\author{
Jan Stanisław Bukowski', Martyna R. Marczuk², Marcin Dziekiewicz'1, Aleksandra Banaszkiewicz \\ 'Department of Paediatric Gastroenterology and Nutrition, Medical University of Warsaw, Warsaw, Poland \\ ${ }^{2}$ Students' Scientific Group GEKON, Department of Paediatric Gastroenterology and Nutrition, Medical University of Warsaw, \\ Warsaw, Poland
}

\section{ABSTRACT}

The aim of this systematic review is to summarise the evidence on the use of endoscopic ductal stenting in children with grade III pancreatic injury (according to the American Association for the Surgery of Trauma AAST). To our review we included all patients $\leq 18$ years old with endoscopic stenting due to an AAST grade III pancreatic injury from a systematic search and a retrospective chart review in our institution. In total, 34 cases of children were evaluated. In 25 cases (73.5\%), the stent was placed across the duct transection site and in seven patients $(20.6 \%)$ it was a transpapillary stent. Endoscopic stent insertion allowed surgery to be avoided in $79.4 \%$ of them. Pseudocysts appeared in $8 / 34$ cases (23.5\%). Endoscopic stenting appears to be a promising treatment option in the majority of children with AAST grade III pancreatic injury; more research is needed.

\section{KEY WORDS:}

ERCP, endoscopic retrograde cholangiopancreatography, paediatric population, AAST, pancreatic trauma.

\section{INTRODUCTION}

Pancreatic injury is rare in children. Reported rates of pancreatic injuries range from $0.12 \%$ to $12 \%$ of all injuries in the paediatric population. Usually, pancreatic injuries occur after a blunt abdominal trauma, most often in boys after a handlebar-related injury [1-5]. Whereas the majority of clinicians accept the idea of a non-surgical treatment for minor pancreatic injuries (grade I and II according to the American Association for the Surgery of Trauma - AAST) and surgical treatment for AAST IV and V injuries, there is a lack of consensus about the management of distal pancreatic duct disruption (grade III by AAST) (Table 1).

Endoscopic retrograde cholangiopancreatography (ERCP), which has been used in adults to diagnose and
TABLE 1. The American Association for the Surgery of Trauma pancreas injury scale

\begin{tabular}{|l|c|c|}
\hline Grade & Type of injury & Description of injury \\
\hline I & Haematoma & Minor contusion without duct injury \\
\cline { 2 - 3 } & Laceration & Superficial laceration without duct injury \\
\hline II & Haematoma & $\begin{array}{c}\text { Major contusion without duct injury } \\
\text { or tissue loss }\end{array}$ \\
\cline { 2 - 3 } III & Laceration & $\begin{array}{c}\text { Major laceration without duct injury } \\
\text { or tissue loss }\end{array}$ \\
\hline IV & Laceration & $\begin{array}{r}\text { Proximal transection or parenchymal } \\
\text { injury with duct injury } \\
\text { injury involving or parenchymal ampulla }\end{array}$ \\
\hline V & Laceration & Massive disruption of pancreatic head \\
\hline
\end{tabular}

\section{ADDRESS FOR CORRESPONDENCE:}

Jan Stanisław Bukowski, Department of Paediatric Gastroenterology and Nutrition, Medical University of Warsaw, 63A Żwirki i Wigury St., 02-091 Warsaw, Poland, ORCID: 0000-0003-3113-5808, e-mail: bukowski.janek@gmail.com 
treat main pancreatic duct injuries by stenting and endoscopic drainage, is still not popular in paediatric departments $[2,3,5]$.

The advantages of therapeutic ERCP include the avoidance of a major abdominal operation with potential risk to the spleen $[2,3]$, the elimination of pseudocyst development from continuing pancreatitis [3], and the avoidance of pancreatic fistulae or small bowel obstruction [6].

On the other hand, non-operative management of pancreatic duct injuries may lead to atrophy of the distal pancreas. Moreover, there is an increased risk for both main pancreatic duct stenosis and post-ERCP pancreatitis [7-9].

The data regarding endoscopic treatment of pancreatic injury in children are very limited and mainly originate from adult case reports and case series. Therefore, we performed this systematic review to summarise the evidence on the use of endoscopic ductal stenting in children with AAST grade III pancreatic injury.

\section{MATERIAL AND METHODS}

A systematic search was independently conducted by two of the authors in parallel using Medline, Embase, and the Cochrane Library (all records up to July 2019). The query list was constructed by using a combination of the following keywords: "child" AND "pancreas" AND ("injury” OR "trauma”) AND ("endoscopy” OR "endoscopic retrograde cholangiopancreatography") AND ("stent” OR "drainage"). Only full-text articles published in English were considered. Reference lists from all of the articles were manually searched to identify further relevant articles. All records using ERCP stenting due to an AAST grade III pancreatic injury in patients $\leq 18$ years old were included in the review. Additionally, a retrospective chart review of all patients with pancreatic injury hospitalised in our institution was conducted.

\section{RESULTS}

The primary search of the databases yielded 129 articles. In total, 15 articles met our inclusion criteria, of which 13 were derived from the primary search and two were derived from the manual reference check. All the articles were published between 2001 and 2018 and described 32 cases of children with a pancreatic trauma treated endoscopically with pancreatic duct stenting. As a result of the retrospective chart review from our institution, we added another two cases. In all cases, the pancreatic injury was assessed as AAST grade III or (in cases in which there was no grading) localised in the body or the tail of the pancreas.

In total, we included 13 boys and 6 girls (in 15 of the cases, sex was not mentioned) between 3 and 18 years of age. Nine patients had a handlebar-related injury. These patients presented with abdominal pain (8/34), vomiting (4/34), and abdominal tenderness (8/34). In all cases, an increased level of amylase or lipase was observed (amylase: range 120-1835 U/l, lipase: range 844-6560 U/l). The time from injury to ERCP ranged between 8 hours and 43 days post injury. Overall, endoscopic stent insertion allowed surgery to be avoided in $78.4 \%$ (27/34) of children. Table 2 presents the detailed characteristics of the children.

In 25 cases (73.5\%), the stent was placed across the duct transection site. In two patients (5.9\%) the stent was described as "stent into collection" and in one case (2.9\%) as "stent almost to the place of disruption". Transpapillary stent insertion was managed in seven cases (20.6\%). Sphincterotomy was performed in 14 patients (41.2\%). Final stent removal was performed between 11 days and 19 months post injury. Only seven children (20.6\%), despite stenting, required a surgical intervention that involved resection of the pancreatic tail or of the tail and body. Pancreatic duct strictures were observed in four patients (11.8\%). Pseudocysts appeared in 8/34 cases (23.5\%): in two cases percutaneous drainage was needed $[10,11]$, and in another two cases cysto-duodenostomy or cysto-gastrostomy was performed $[2,8]$. In the remaining pseudocyst cases, a transpapillary stent was inserted in a 14-year-old patient who eventually required surgery [5], two patients were treated conservatively $[5,12]$, and one case had no information about the management of the pseudocyst [5].

\section{DISCUSSION}

This is the first systematic review summarising AAST grade III pancreatic injuries treated with endoscopic stent placement in children.

Because there has been no systematic review analysing pancreatic duct stenting in both children and adults with only AAST grade III pancreatic injury, the present results can only be compared with small, mostly singlecentre case series published previously.

Although the data are very limited, it seems that in all these case series, the level of avoiding surgical necessity, was high.

In 2015, Garvey et al. reported on nine patients with pancreatic injuries following abdominal blunt trauma, who had undergone ERCP. Six of these patients were classified as having AAST grade III pancreatic injuries. In two of these patients, stents were successfully inserted into the main pancreatic duct and allowed to avoid surgery. In the remaining four patients, ERCP was carried out without stenting, and two required distal pancreatectomy [13].

Houben et al. summarised their findings of main pancreatic duct injuries in 15 children. Four of them presented with grade III pancreatic injury. Three of the children were managed with endoscopic stent insertion - two stents into the collection and one across the duct. The patient with the stent across the duct also required an endoscopic cystgastrostomy due to pseudocyst formation. The fourth patient was treated without any intervention [2].

Rosenfeld et al. analysed the results of 26 patients from 22 paediatric trauma centres. Of the 26 patients, 


\begin{tabular}{|c|c|c|c|c|c|c|c|}
\hline \multirow[b]{2}{*}{ 产 } & \multirow[b]{2}{*}{$\approx$} & \multirow{2}{*}{ 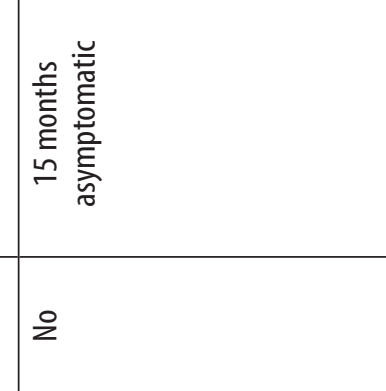 } & \multirow{2}{*}{ 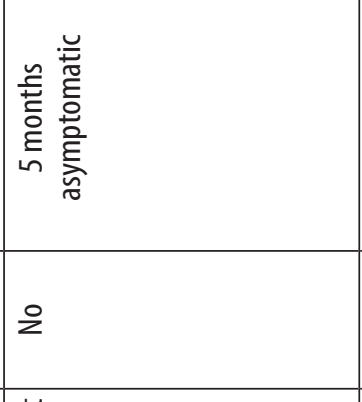 } & \multicolumn{3}{|c|}{ 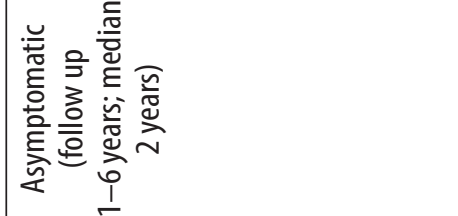 } & \multirow{2}{*}{ 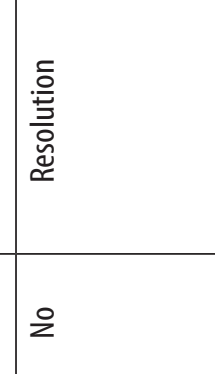 } \\
\hline & & & & 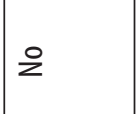 & $\stackrel{2}{2}$ & $\stackrel{2}{2}$ & \\
\hline 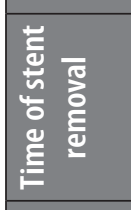 & 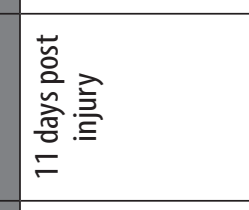 & 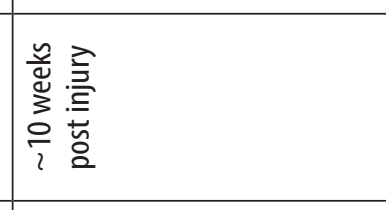 & 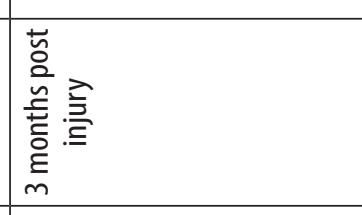 & \multicolumn{3}{|l|}{ 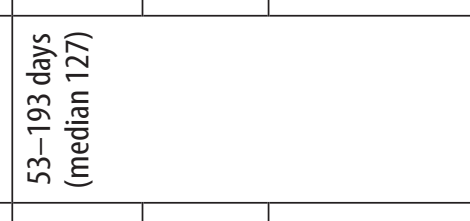 } & 1 \\
\hline 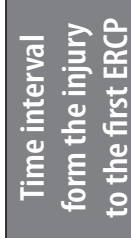 & 资 & $\begin{array}{l}\frac{2}{3} \\
\frac{3}{2} \\
\frac{3}{2}\end{array}$ & 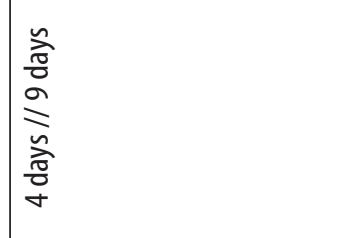 & 瓷 & 登 & 毬 & 1 \\
\hline & 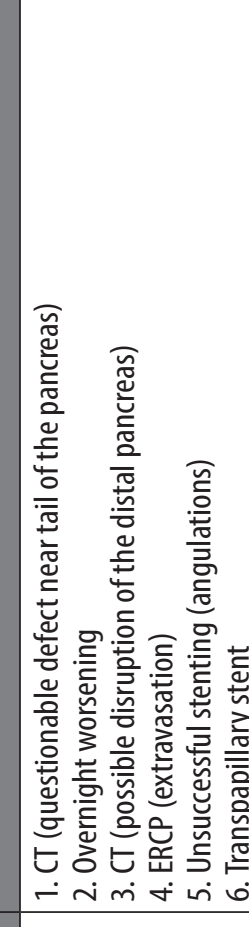 & 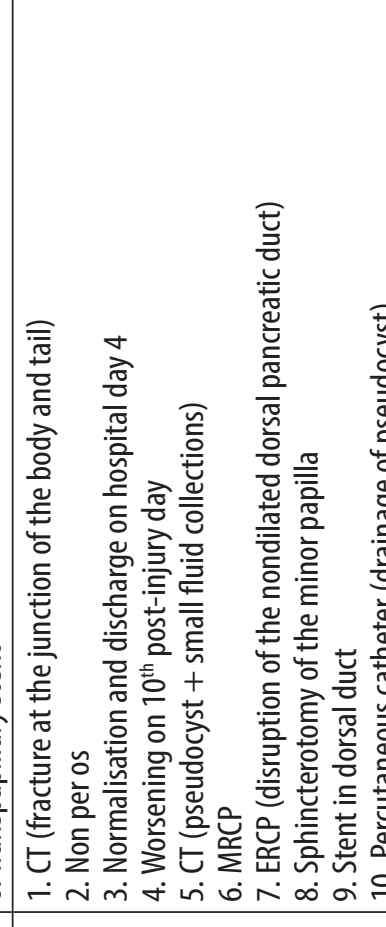 & 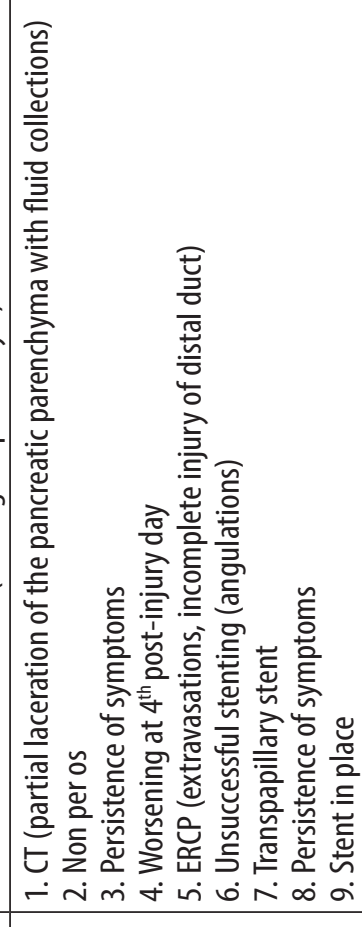 & 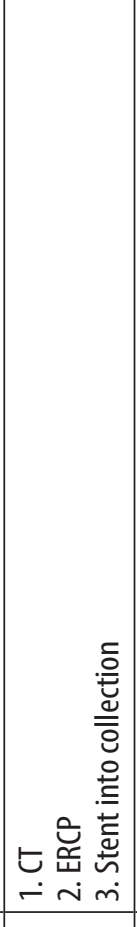 & 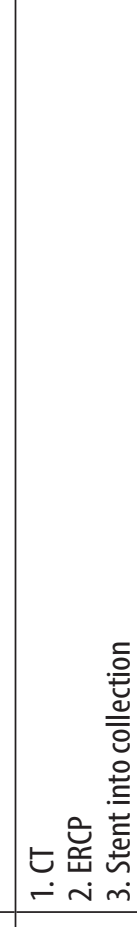 & 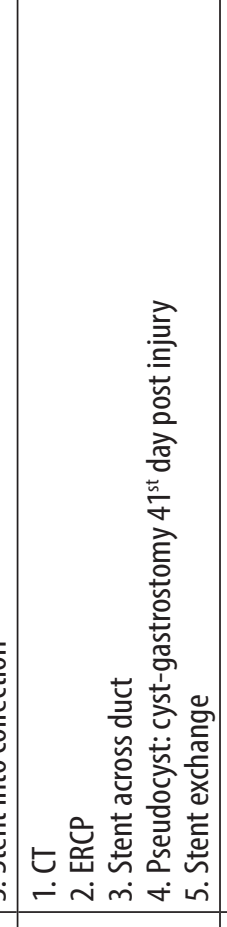 & | \\
\hline 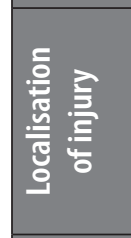 & 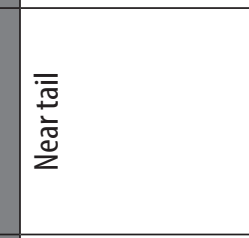 & 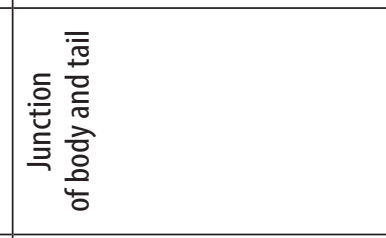 & 高 & 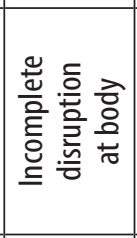 & 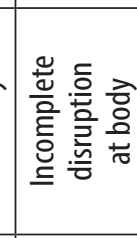 & 㐔 & 홍 \\
\hline 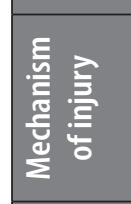 & 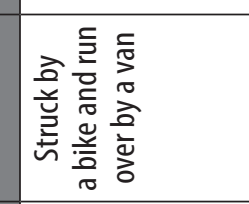 & 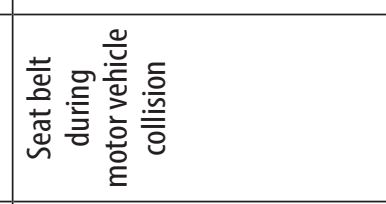 & 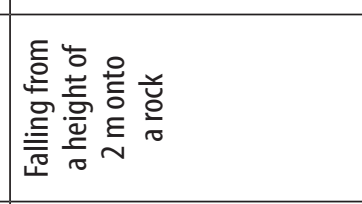 & 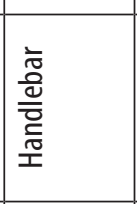 & 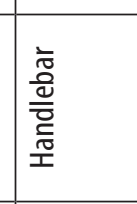 & 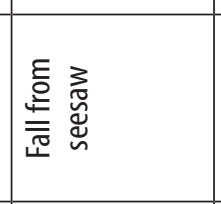 & 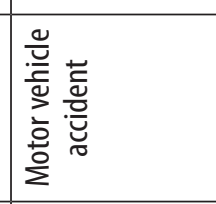 \\
\hline 害高 & $\infty$ & $=$ & $=$ & $\infty$ & $\cong$ & $=$ & 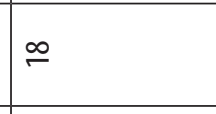 \\
\hline 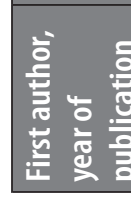 & $\overrightarrow{0}$ & 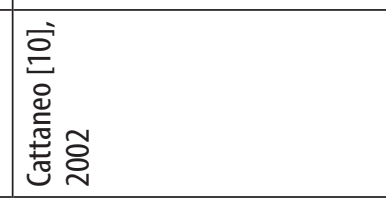 & $\begin{array}{l}\text { 离 } \\
\text { 总宫 }\end{array}$ & 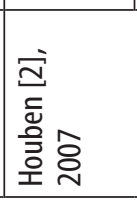 & & & 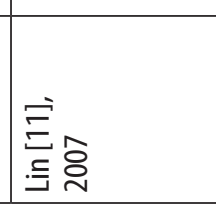 \\
\hline
\end{tabular}




\begin{tabular}{|c|c|c|c|c|c|c|}
\hline 套 & 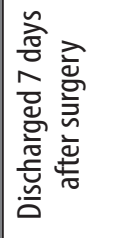 & 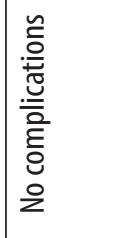 & 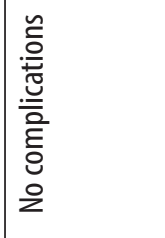 & 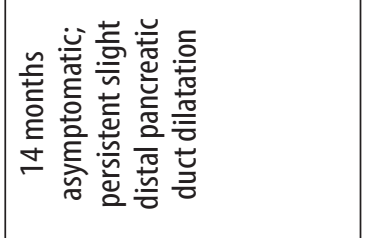 & 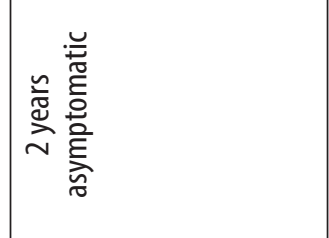 & 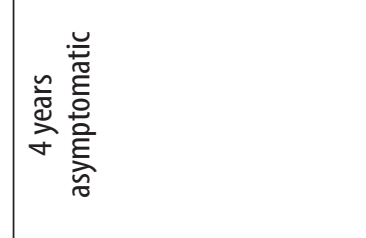 \\
\hline 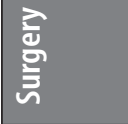 & 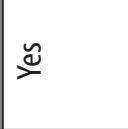 & $\stackrel{2}{2}$ & $\stackrel{2}{2}$ & $\stackrel{2}{2}$ & 2 & $\stackrel{0}{2}$ \\
\hline 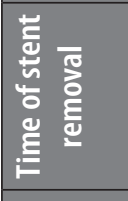 & 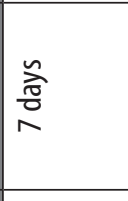 & 1 & 1 & 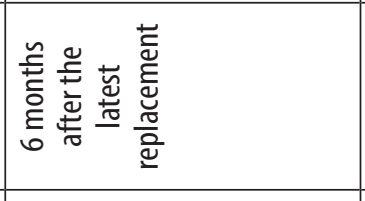 & 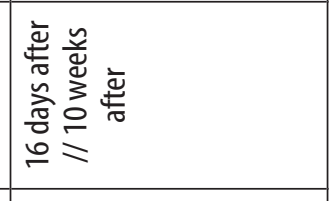 & 1 \\
\hline 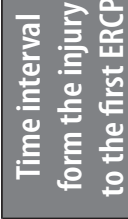 & 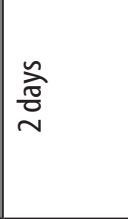 & 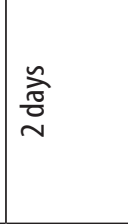 & 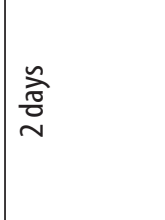 & 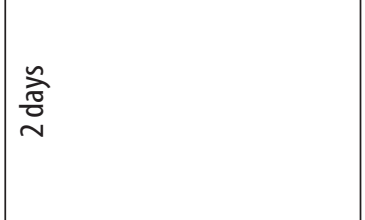 & 1 & 1 \\
\hline 厸 & 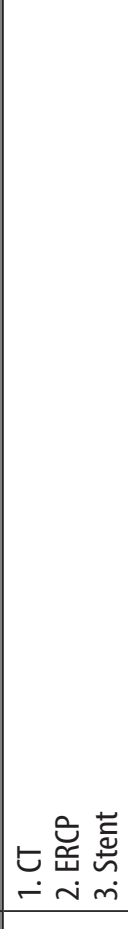 & 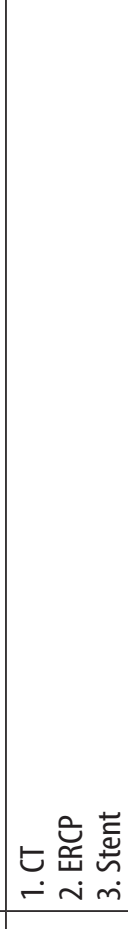 & 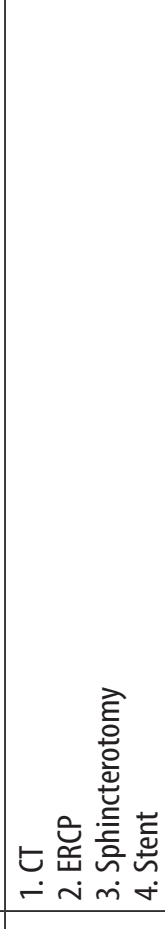 & 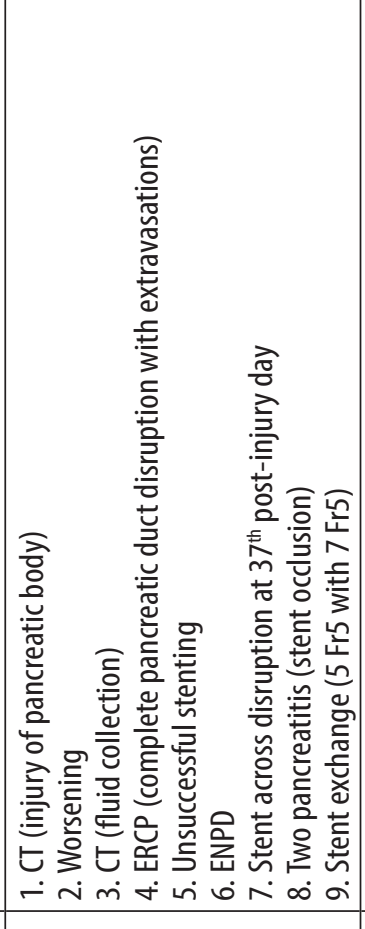 & 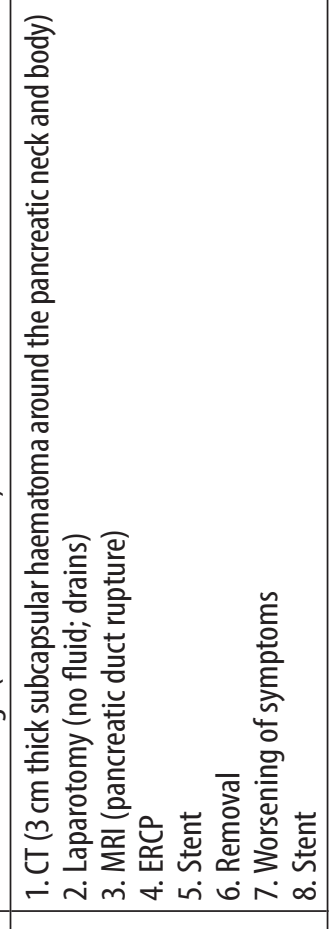 & 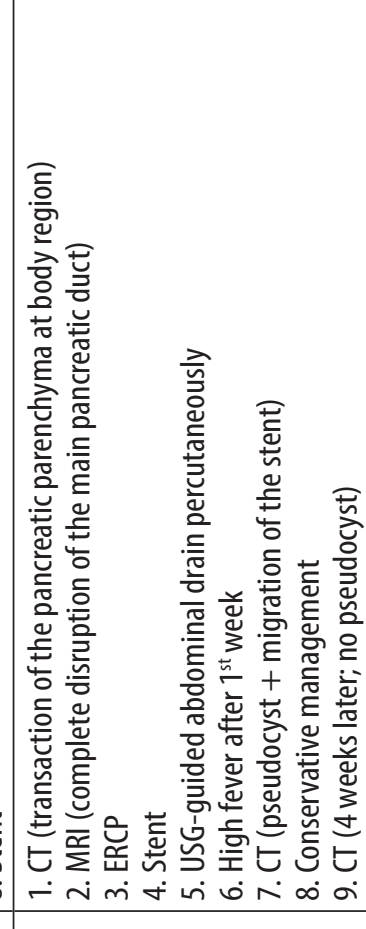 \\
\hline 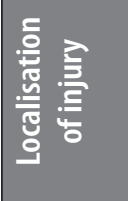 & $\overline{\overline{1}}$ & 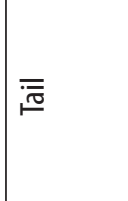 & 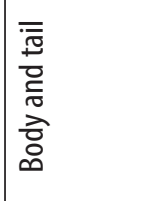 & 흠 & 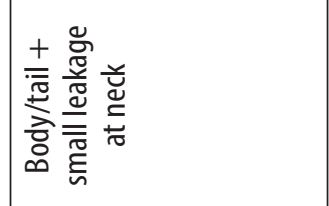 & 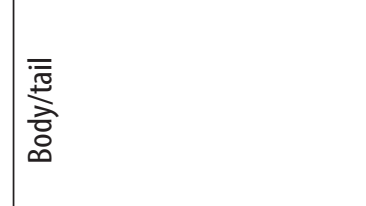 \\
\hline 焉 & 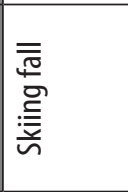 & 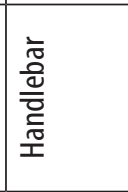 & 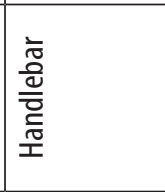 & 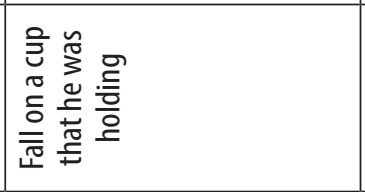 & 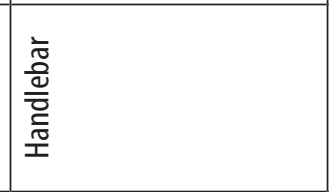 & 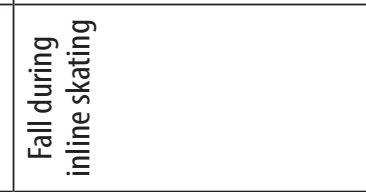 \\
\hline 离啇 & 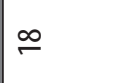 & $\infty$ & $a$ & $\infty$ & $=$ & $\circ$ \\
\hline 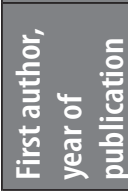 & 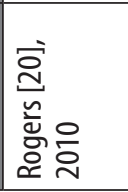 & \multicolumn{2}{|l|}{ 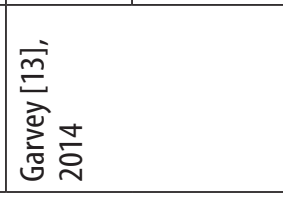 } & 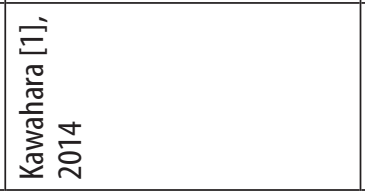 & \multicolumn{2}{|l|}{ 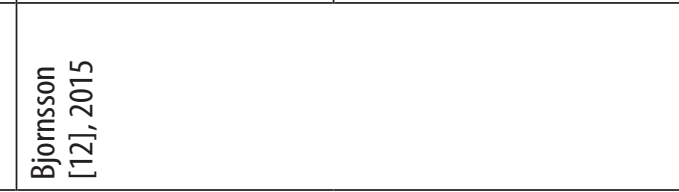 } \\
\hline
\end{tabular}




\begin{tabular}{|c|c|c|c|c|c|c|c|c|c|}
\hline 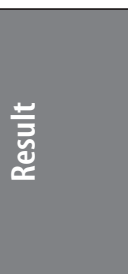 & 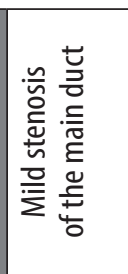 & 1 & 1 & 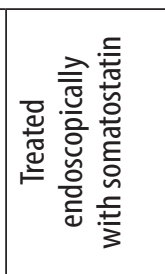 & 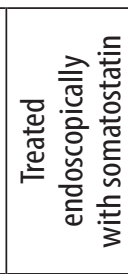 & 1 & 1 & 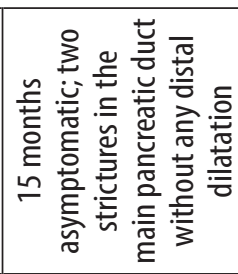 & 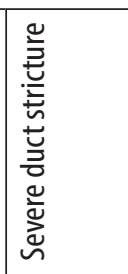 \\
\hline 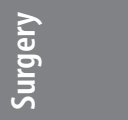 & $\stackrel{2}{2}$ & $\stackrel{0}{\beth}$ & 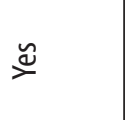 & ' & ' & 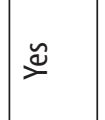 & $\cong$ & $\stackrel{2}{2}$ & $\stackrel{2}{2}$ \\
\hline 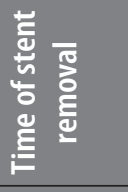 & 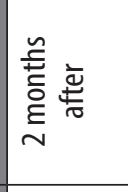 & 1 & 1 & 㙜 & 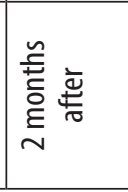 & 1 & 1 & 1 & 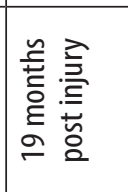 \\
\hline 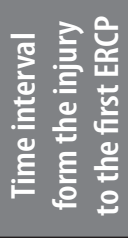 & & \multicolumn{6}{|c|}{ 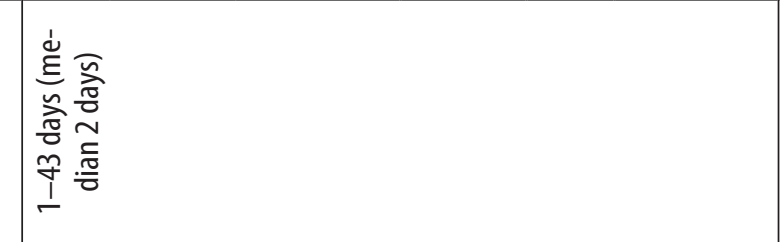 } & 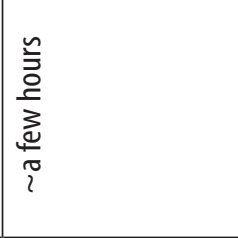 & 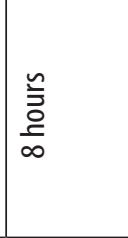 \\
\hline 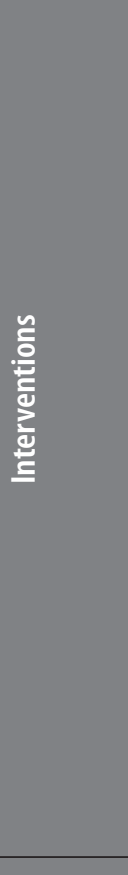 & 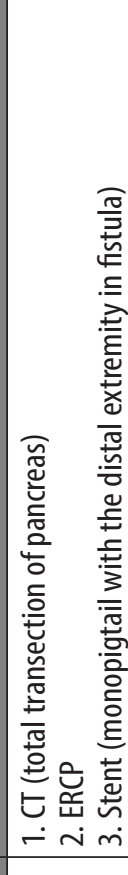 & \multicolumn{6}{|c|}{ 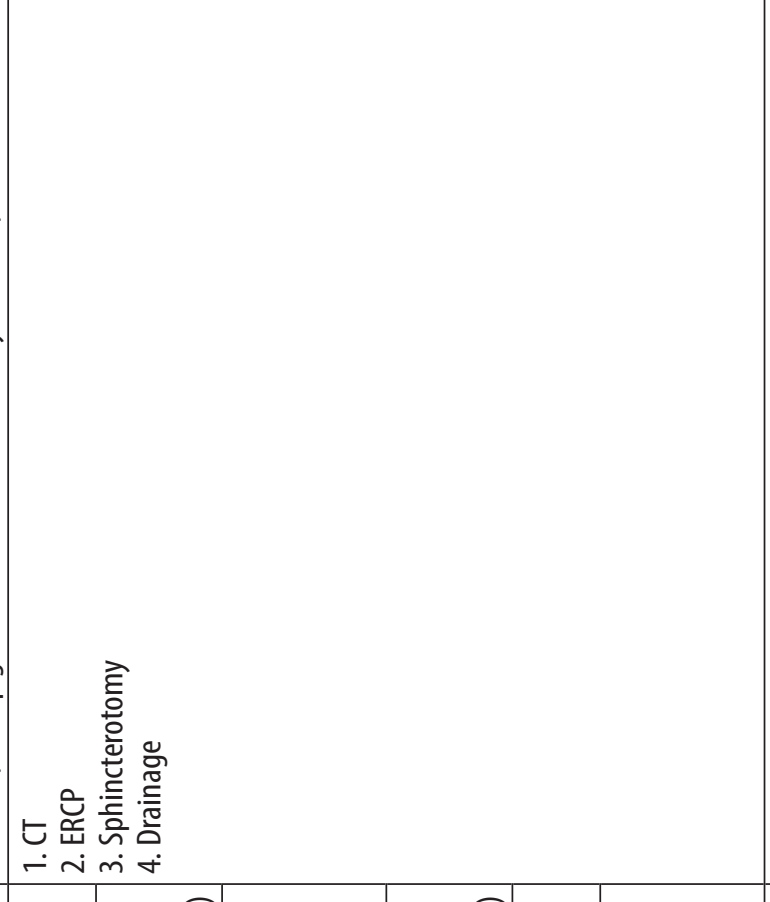 } & 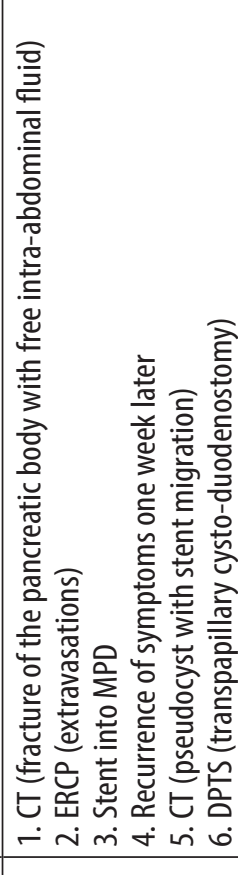 & 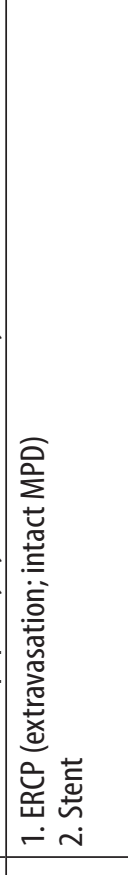 \\
\hline 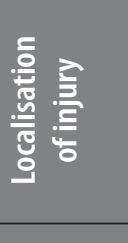 & 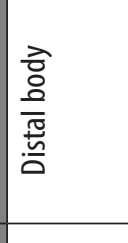 & 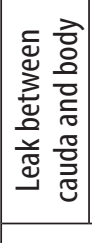 & 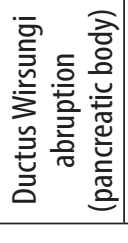 & 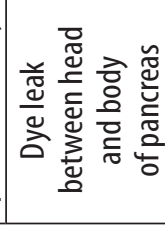 & 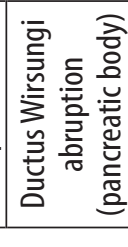 & 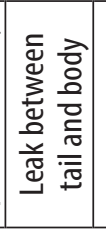 & 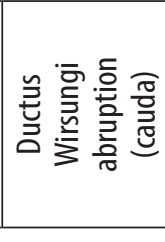 & 흥 & 흥 \\
\hline 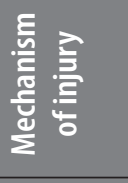 & 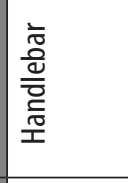 & \multicolumn{6}{|c|}{ 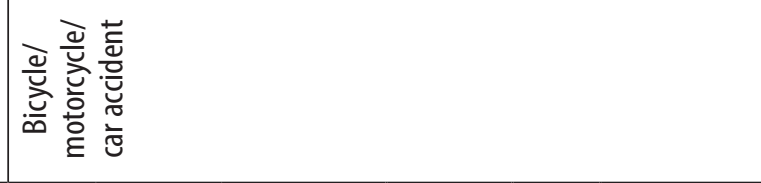 } & 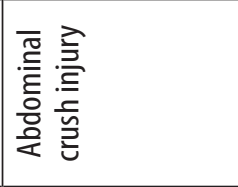 & 壱 \\
\hline 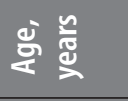 & $=$ & $m$ & $m$ & $\bullet$ & 0 & n & $=$ & in & 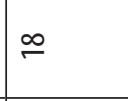 \\
\hline 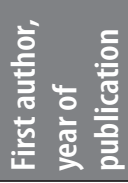 & 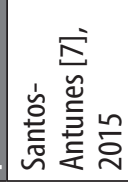 & \multicolumn{6}{|c|}{ 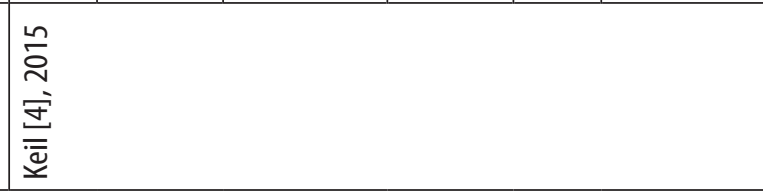 } & 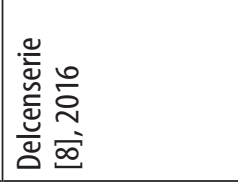 & 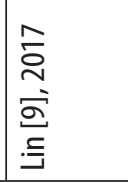 \\
\hline
\end{tabular}




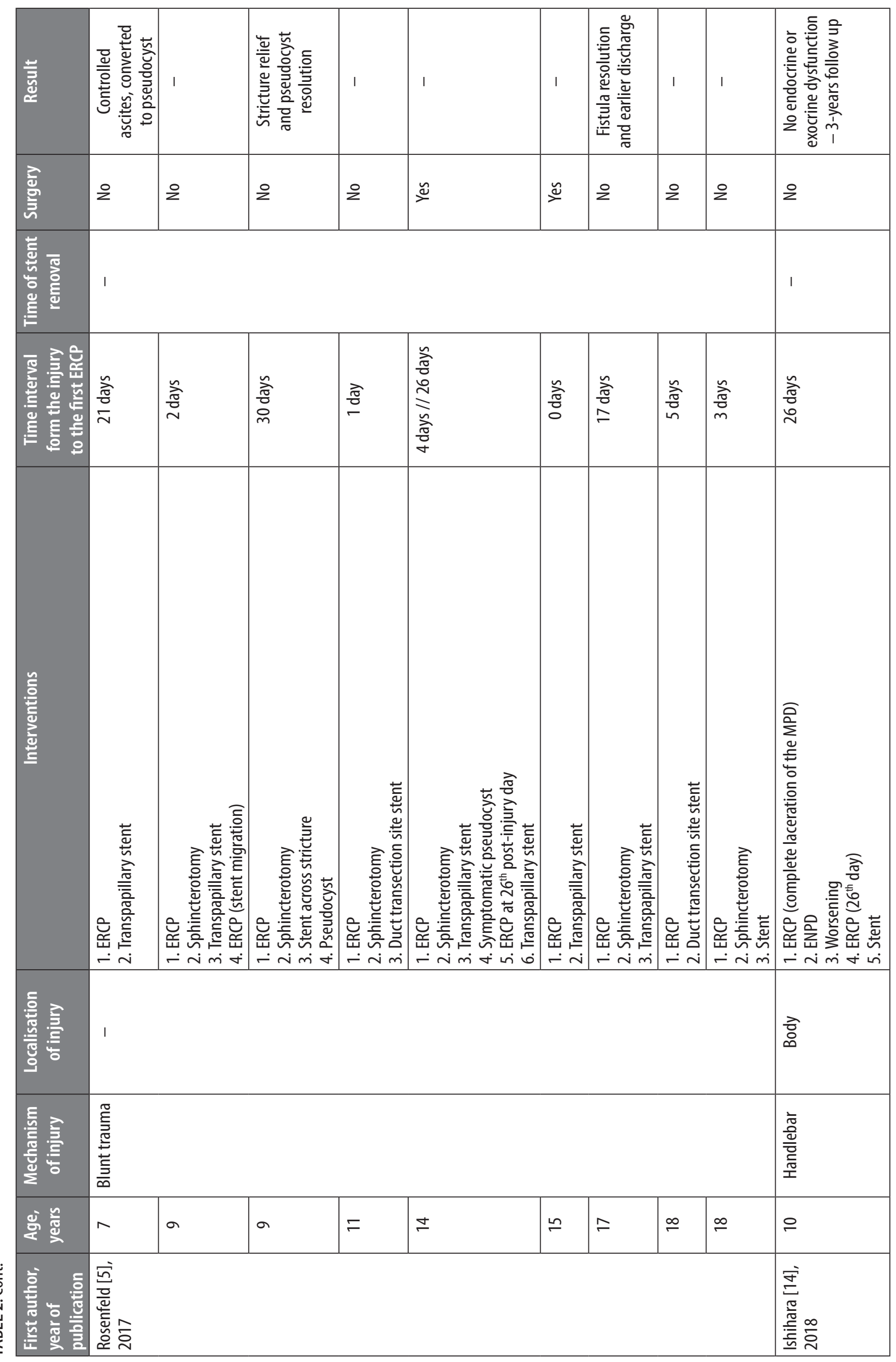




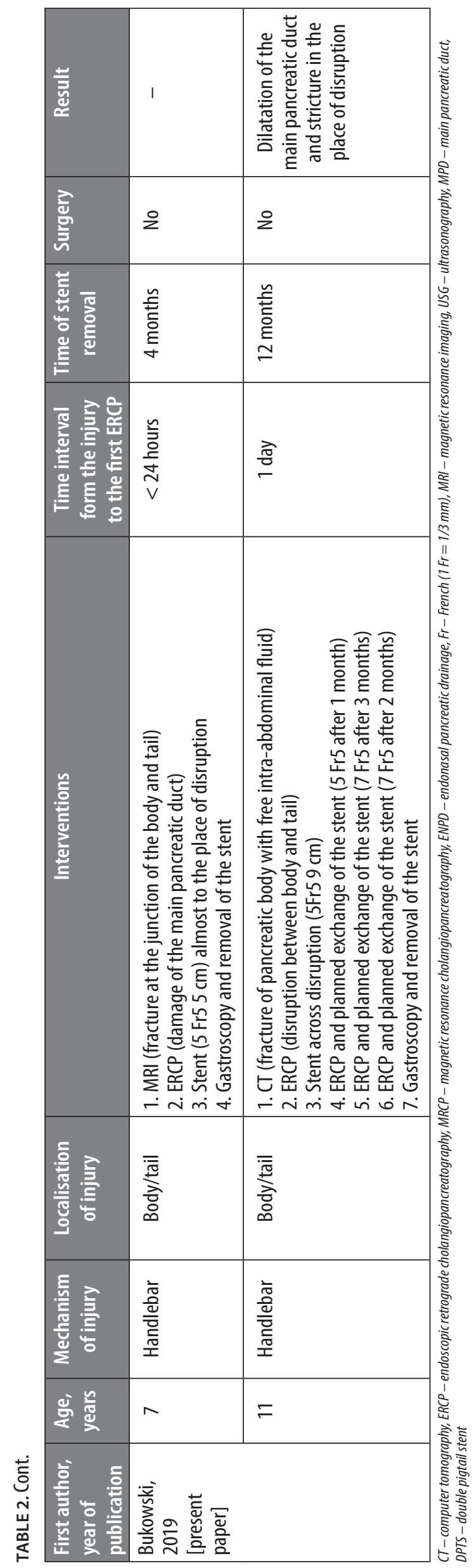

12 were classified as having an AAST grade III pancreatic injury. Five of children had transpapillary stent insertions (two of them also required distal pancreatectomy), three patients had insertions into the place of injury, and one child had a stent insertion across the location of injury. Cannulation of the main pancreatic duct was not possible in one patient, and in the two remaining patients, interventions during ERCP were not attempted and distal pancreatectomy was carried out [5].

Rapid endoscopic intervention is considered one of the most important factors for successful endoscopic management because it means stent insertion can be performed before the highest levels of oedema are reached. In our study, we analysed the time between injury and ERCP. Endoscopic stenting was performed between 8 hours and 43 days post injury, and we did not find any correlation between the success of the stent insertion and the timing of the procedure after injury. Moreover, Houben et al. reported that therapeutic ERCP is technically possible even weeks after injury, and this timing also helps to reduce the need for open surgery [2]. It is important to mark that the stent that is placed shortly after injury should be responsible for minimising extravasation and increasing the chance of a spontaneous successful resolution of the ductal disruption. When it comes to drainage several days or weeks after the injury, the aim is to "rescue" a patient who has failed conservative management by developing a fistula, a cyst, or a stricture.

Our results are also supported by adult data. Bjornsson et al. described 57 adult patients with blunt pancreatic injury treated endoscopically [12]. The treatment allowed surgery to be avoided in all but three patients. The time from injury to stenting ranged from 8 hours to 45 days. Unfortunately, the authors did not perform an AAST grading analysis; therefore, we cannot compare it with our results.

We also tried to analyse seven additional cases of patients who required a surgical intervention despite stenting. However, we cannot elaborate on the profiles of these patients because detailed case data were not provided.

One common complication of pancreatic injury is pseudocyst formation $[4,10]$, which may occur in both endoscopically and conservatively treated patients $[1,2,4,10$, 14]. In our study, despite using ERCP, 7/34 cases (20.6\%) were associated with pseudocyst formation (in one case described by Cattaneo et al. - pseudocyst appeared before ERCP); one of them was treated by surgery. While pseudocyst data from children are scarce, a large study $(n=653)$ by Mohseni et al. in adults with AAST grade III pancreatic injury, describing the outcomes after resection and non-resection management, showed that a pseudocyst was found only in one patient treated non-surgically [15].

The aim of non-operative management of pancreatic duct injury is to insert a stent across the disruption to prevent leakage of pancreatic juice into the retroperitoneal space. However, it is not always technically possible. In 
our review, seven patients underwent transpapillary stent insertion, and only two of them also required distal pancreatectomy. It therefore seems that even if a stent cannot be placed during the first attempt, endoscopic internal drainage (sphincterotomy or transpapillary stent) can still be performed. This drainage results in a reduction of intrapancreatic pressure and may prevent the formation of pancreatic pseudocysts. In these cases, adequate drainage of pancreatic juice and reduced oedema may allow for subsequent definitive intervention [6].

It is difficult to compare our results with the results of adult studies assessing endoscopic stenting after pancreatic injury. First, there are only a few articles on this topic, and second, most of them are case reports [16-19]. Moreover, the data are heterogeneous with the cases being analysed without pancreatic injury grading, without the aetiology being reported (i.e. trauma or acute pancreatitis) and without the details of stent placement (i.e. transpapillary or at an injury site) [20].

The main advantage of our study is that, for the first time, we systematically gathered all the published cases of children with AAST grade III pancreatic injury, who were treated with pancreatic stent placement. Although the data are limited, it seems that this procedure is safe even in young children and allows avoidance of surgery in a high percentage of patients.

Because there is no published prospective study assessing this issue, we hope that this systematic review may be the first step toward planning a multi-centre study to assess the possibility of avoiding surgery by endoscopic stent placement in children who have a main pancreatic duct injury after abdominal trauma. This review also has some limitations. Due to its retrospective character, many important parameters or variables (e.g. time to follow-up) are very heterogeneous or not reported. Moreover, as with any descriptive study, a cause-and-effect relationship cannot be established, and our findings cannot be generalised.

Because of the lack of multicentre, well-prepared studies comparing pancreatic duct stenting with surgery in patients with AAST III grade pancreatic injury we cannot unambiguously admit that endoscopic stenting is superior to the surgical approach. Nevertheless, in some selected cases it may be a good treatment option.

\section{CONCLUSIONS}

In conclusion, in this systematic review, we found endoscopic stent placement to be a promising treatment option in the majority of children with AAST grade III pancreatic trauma. We believe that the results of our study will encourage paediatric gastroenterologists and surgeons to consider treating this group of patients endoscopically.

\section{DISCLOSURE}

The authors declare no conflict of interest.

\section{REFERENCES}

1. Kawahara I, Maeda K, Ono S, et al. Surgical reconstruction and endoscopic pancreatic stent for traumatic pancreatic duct disruption. Pediatr Surg Int 2014; 30: 951-956.

2. Houben $\mathrm{CH}$, Ade-Ajayi N, Patel S, et al. Traumatic pancreatic duct injury in children: minimally invasive approach to management. J Pediatr Surg 2007; 42: 629-635.

3. Canty TG, Weinman D. Treatment of pancreatic duct disruption in children by an endoscopically placed stent. J Pediatr Surg 2001; 36: 345-348.

4. Keil R, Drabek J, Lochmannova J, et al. What is the role of endoscopic retrograde cholangiopancreatography in assessing traumatic rupture of the pancreatic in children? Scand J Gastroenterol 2015; 51: 218-224.

5. Rosenfeld EH, Vogel AM, Klinkner DB, et al. The utility of ERCP in pediatric pancreatic trauma. J Pediatr Surg 2018; 53: 146-151.

6. Meier DE, Dale-Coln C, Haicks BA, et al. Early operation in children with pancreas transection. J Pediatr Surg 2001; 36: 341-344.

7. Santos-Antunes J, Pereira P, Rodrigues-Pinto E, et al. Successful management of traumatic pancreatic transection using ERCP-guided pancreatic stenting. Endoscopy 2015; 47: E112-113.

8. Delcenserie R, Ricard J, Yzet T, et al. Conservative endoscopic management for pancreatic trauma. J Visc Surg 2016; 153: 391-394.

9. Lin BC, Wong YC, Chen RJ, et al. Major pancreatic duct continuity is the crucial determinant in the management of blunt pancreatic injury: a pancreatographic classification. Surg Endosc 2017; 31: 4201-4210.

10. Cattaneo SM, Sedlack JD, Kalloo AN, et al. Management of a pancreatic duct injury with an endoscopically placed stent. Surgery 2004; 135: 690-692.

11. Lin BC, Fang JF, Wong YC, et al. Blunt pancreatic trauma and pseudocyst: management of major pancreatic duct injury. Injury 2007; 38: 588-593.

12. Bjornsson B, Kullman E, Gasslander T, et al. Early endoscopic treatment of blunt traumatic pancreatic injury. Scand J Gastroenterol 2015; 50: 1435-1443.

13. Garvey EM, Haakinson DJ, McOmber M, et al. Role of ERCP in pediatric blunt abdominal trauma: a case series at a level one pediatric trauma center. J Pediatr Surg 2015; 50: 335-338.

14. Ishihara $T$, Nishiyama $K, A b e T$, et al. Efficacy of stent and drainage for blunt pancreatic injury. J Pediatr Surg 2018; 30: 82-85.

15. Mohseni S, Holzmacher J, Sjolina G, et al. Outcomes after resection versus non-resection management of penetrating grade III and IV pancreatic injury: a trauma quality improvement (TQIP) databank analysis. Injury 2018; 49: 27-32.

16. Kim HS, Lee DK, Kim IW, et al. The role of endoscopic retrograde pancreatography in the treatment of traumatic pancreatic duct injury. Gastrointest Endosc 2001; 54: 49-55.

17. Telford JJ, Farrell JJ, Saltzman JR, et al. Pancreatic stent placement for duct disruption. Gastrointest Endosc 2002; 56: 18-24.

18. Thomson DA, Krige JE, Thomson SR, et al. The role of endoscopic retrograde pancreatography in pancreatic trauma: a critical appraisal of 48 patients treated at tertiary institution. J Trauma Acute Care Surg 2014; 76: 1362-1366.

19. Varadarajulu S, Noone TC, Tutuian R, et al. Predictors of outcome in pancreatic dust disruption managed by endoscopic transpapillary stent placement. Gastrointest Endosc 2005; 61: 568-575.

20. Rogers SJ, Cello JP, Schecter WP. Endoscopic retrograde cholangiopancreatography in patients with pancreatic trauma. J Trauma 2010; 68: 538-544.

21. Cay A, Imamoglu M, Bektas O, et al. Nonoperative treatment of traumatic pancreatic duct disruption in children with an endoscopically placed stent. J Pediatr Surg 2005; 40: 9-12. 